\title{
Covariant realizations of kappa-deformed space
}

\author{
Stjepan Meljanac a 1, Saša Krešić-Jurić ${ }^{b}: 2$ and Marko Stojić ${ }^{a} \sqrt[3]{3}$ \\ ${ }^{a}$ Rudjer Bošković Institute, Bijenička c. 54, 10002 Zagreb, Croatia \\ ${ }^{b}$ Faculty of Natural and Mathematical Sciences, Teslina 12, 21000 Split, Croatia
}

\begin{abstract}
We study a Lie algebra type $\kappa$-deformed space with undeformed rotation algebra and commutative vector-like Dirac derivatives in a covariant way. Space deformation depends on an arbitrary vector. Infinitely many covariant realizations in terms of commuting coordinates of undeformed space and their derivatives are constructed. The corresponding coproducts and star products are found and related in a new way. All covariant realizations are physically equivalent. Specially, a few simple realizations are found and discussed. The scalar fields, invariants and the notion of invariant integration is discussed in the natural realization.

PACS number(s):
\end{abstract}

Keywords: noncommutative space, covariant realizations, field theory.

\footnotetext{
${ }^{1}$ e-mail: meljanac@thphys.irb.hr

${ }^{2}$ e-mail: skresic@fesb.hr

${ }^{3}$ e-mail: marko.stojic@zg.htnet.hr
} 


\section{Introduction}

In the last decade there has been a growing interest in the formulation of physical theories defined on noncommutative (NC) spaces. Consistency of such theories and their implications were studied in [1]-[5]. It is important to classify $\mathrm{NC}$ spaces and investigate their properties, and particularly to develop a unifying approach to a generalized theory for such spaces that are convenient for physical applications. The notion of generalized symmetries and their role in the analysis of NC spaces is also crucial. In order to make a contribution in this direction, we analyze a NC space of the Lie algebra type, in particular the so-called $\kappa$-deformed space introduced in [6]-[8].

For simplicity we restrict our attention to $\kappa$-deformed Euclidean space, although the analysis can be easily extended to $\kappa$-deformed Minkowski space. The noncommutativity of coordinates depends on a deformation parameter which is an arbitrary vector $a \in \mathbb{R}^{n}$. The dimensional parameter $|a|=1 / \kappa$ has a very small length which yields the undeformed Euclidean space in the limit as $|a| \rightarrow 0$. The NC coordinates and the generators of generalized rotations form an extended Lie algebra. The subalgebra formed by the rotation generators is the ordinary $S O_{a}(n)$ algebra, i.e. the ordinary Lorentz algebra in the case of $\kappa$-deformed Minkowski space. Dirac derivatives are assumed to commute mutually and transform as a vector representation under $S O_{a}(n)$ algebra. This $\kappa$-deformed space was studied by different groups, from both the mathematical and physical point of view [9]-[27]. There is also an interesting connection to the Doubly Special Relativity program [15], 16]. Realizations of NC spaces in terms of commutative coordinates and derivative operators were obtained and discussed in the cases of symmetric ordering and normal (left and right) ordering of NC coordinates [13, 21]. An infinite family of noncovariant realizations was found in 22 . Although a single space may be realized in many different ways, physical results do not depend on concrete realizations, i.e. orderings (see also [24]).

Our motivation in this paper is to construct covariant realizations for general $\kappa$-deformed Euclidean spaces depending on an arbitrary deformation vector $a$. We analyze such spaces by using the methods developed for deformed single and multimode oscillators in the Fock space representations [29]-[36]. In particular, we use the methods for constructing deformed creation and annihilation operators in terms 
of ordinary bosonic multimode oscillators, i.e. a kind of bosonization [29], [30], 335]. Also, we employ the construction of transition number operators and, in general, of generators proposed in [30], [31], [34].

The simple connection between the creation and annihilation operators with NC coordinates and Dirac derivatives is established by using the Bargman type representation. We find infinitely many new covariant realizations in terms of commutative coordinates and derivative operators. The realizations depend on certain parameter functions, but they are all on an equal footing and the physical results do not depend on them. For a special choice of the parameter functions we obtain some particularly simple realizations: covariant left, right and natural realizations. These realizations are considered in detail, and a coproduct and star product are associated to each of them.

The outline of the paper is as follows. In Sect. 2 we introduce a Lie algebra type of $\kappa$-deformed Euclidean space. We also define the rotation algebra $S O_{a}(n)$ which is compatible with $\kappa$-deformations, and introduce the Dirac derivative and the Laplace operator. The shift operator which plays an important role in describing the algebra generated by $\mathrm{NC}$ coordinates, the rotation generators and the Dirac derivative are also introduced. Special consideration is given to the generalized Leibniz's rule and coproduct. We derive expressions for the coproduct of the rotation generators and the Dirac derivative, and of the left and right deformations of the ordinary derivative. Sect. 3 deals with covariant realizations of the $\kappa$-deformed Euclidean space and the operators introduced in Sect. 2. We find an infinite family of covariant realizations in terms of commutative coordinates and derivative operators. In particular, we obtain two types of realizations (type I and type II) which depend on an arbitrary parameter function $\varphi$. For special choices of $\varphi$, we construct particularly simple covariant realizations: left, right, symmetric and natural realizations. In Sect. 4 we consider the star product for the realizations discussed in Sect. 3. A general formula for the star product is given to second order in the deformation parameter $a$, and closed form expressions are obtained in the left, right and symmetric realizations. We also introduce the notion of equivalent star products using similarity transformations. We show that any star product in the realization of type I can be obtained from the star product in the right realization using similarity transformations. In subsection 4.4 we introduce scalar fields in $\mathrm{NC}$ coordinates and demonstrate their 
simple properties in terms of the natural realization. Constructions of invariants and invariant integration on $\mathrm{NC}$ spaces are also presented. Finally, in Sect. 5 a short conclusion is given.

\section{Kappa-deformed Euclidean space}

Consider a Lie algebra type noncommutative (NC) space generated by coordinates $\hat{x}_{1}, \hat{x}_{2}, \ldots, \hat{x}_{n}$ satisfying the commutation relations

$$
\left[\hat{x}_{\mu}, \hat{x}_{\nu}\right]=i\left(a_{\mu} \hat{x}_{\nu}-a_{\nu} \hat{x}_{\mu}\right)
$$

where $\mu, \nu=1, \ldots, n$ and $a_{1}, a_{2}, \ldots, a_{n}$ are componenets of a vector $a \in \mathbb{R}^{n}$ which describes a deformation of the Euclidean space [19]-[22]. The structure constants are given by

$$
C_{\mu \nu \lambda}=a_{\mu} \delta_{\nu \lambda}-a_{\nu} \delta_{\mu \lambda}
$$

In the limit as $a \rightarrow 0$, we have $\hat{x}_{\mu} \rightarrow x_{\mu}$, the ordinary commutative coordinates.

Let $S O_{a}(n)$ be the ordinary rotation algebra with generators $M_{\mu \nu}$ satisfying

$$
\begin{aligned}
M_{\mu \nu} & =-M_{\nu \mu} \\
{\left[M_{\mu \nu}, M_{\lambda \rho}\right] } & =\delta_{\nu \lambda} M_{\mu \rho}-\delta_{\mu \lambda} M_{\nu \rho}-\delta_{\nu \rho} M_{\mu \lambda}+\delta_{\mu \rho} M_{\nu \lambda} .
\end{aligned}
$$

We require that the rotation generators $M_{\mu \nu}$ and the coordinates $\hat{x}_{\mu}$ form an extended Lie algebra. The most general form of the commutator $\left[M_{\mu \nu}, \hat{x}_{\lambda}\right]$ must be linear in $M_{\mu \nu}$ and $\hat{x}_{\lambda}$, antisymmetric in the indices $\mu$ and $\nu$, and with the smooth limit $\left[M_{\mu \nu}, \hat{x}_{\lambda}\right] \rightarrow x_{\mu} \delta_{\nu \lambda}-x_{\nu} \delta_{\mu \lambda}$ as $a \rightarrow 0$. The required form is given by

$$
\begin{aligned}
{\left[M_{\mu \nu}, \hat{x}_{\lambda}\right] } & =\hat{x}_{\mu} \delta_{\nu \lambda}-\hat{x}_{\nu} \delta_{\mu \lambda} \\
& +i s a_{\lambda} M_{\mu \nu}-i t\left(a_{\mu} M_{\nu \lambda}-a_{\nu} M_{\mu \lambda}\right)+i u a_{\alpha}\left(M_{\alpha \mu} \delta_{\nu \lambda}-M_{\alpha \nu} \delta_{\mu \lambda}\right)
\end{aligned}
$$

for some $s, t, u \in \mathbb{R}$, where summation over repeated indices in assumed. The necessary and sufficient condition for consistency of the extended Lie algebra is that the Jacobi identity holds for all combinations of the generators $M_{\mu \nu}$ and $\hat{x}_{\lambda}$. One can verify that this is satisfied for the unique values of the parameters $s=u=0$ and $t=1[22$. Hence,

$$
\left[M_{\mu \nu}, \hat{x}_{\lambda}\right]=\hat{x}_{\mu} \delta_{\nu \lambda}-\hat{x}_{\nu} \delta_{\mu \lambda}-i\left(a_{\mu} M_{\nu \lambda}-a_{\nu} M_{\mu \lambda}\right)
$$


Having introduced the rotation algebra $S O_{a}(n)$, it is natural to consider the Dirac derivatives $D_{\mu}$ satisfying

$$
\begin{aligned}
{\left[D_{\mu}, D_{\nu}\right] } & =0, \\
{\left[M_{\mu \nu}, D_{\lambda}\right] } & =\delta_{\nu \lambda} D_{\mu}-\delta_{\mu \lambda} D_{\nu} .
\end{aligned}
$$

The generators $M_{\mu}$ and $D_{\lambda}$ form the undeformed $I S O_{a}(n)$ algebra, i.e. Poincaré algebra in the case of $\kappa$-deformed Minkowski space. Note that the operator $D^{2}=$ $D_{\mu} D_{\mu}$ is invariant under rotations since $\left[M_{\mu \nu}, D^{2}\right]=0$.

We also wish to define commutation relations for $D_{\mu}$ and $\hat{x}_{\nu}$. The consistency condition is that the Jacobi identity is satisfied for all combinations of the generators $M_{\mu \nu}, D_{\lambda}$ and $\hat{x}_{\rho}$. It can be shown [22] that the correct form of the commutator is given by

$$
\left[D_{\mu}, \hat{x}_{\nu}\right]=\delta_{\mu \nu} \sqrt{1-a^{2} D^{2}}+i C_{\mu \alpha \nu} D_{\alpha}
$$

The algebra generated by $D_{\mu}$ and $\hat{x}_{\nu}$ is a deformed Heisenberg algebra. We note that, in the limit as $a \rightarrow 0$, the commutation relations (1), (7) and (9) yield the ordinary undeformed Heisenberg algebra. Hence, $D_{\mu} \rightarrow \partial_{\mu}$ and $\hat{x}_{\mu} \rightarrow x_{\mu}$ as $a \rightarrow 0$, where $\partial_{\mu}=\frac{\partial}{\partial x_{\mu}}$. Particularly, in the one-dimensional case $n=1$, Eq. (9) leads to a generalized uncertainty relation with mininal length [37].

A function $f(\hat{x}, D)$, where $f(\hat{x}, D)$ denotes a formal power series in monomials $\hat{x}_{\mu_{1}} \hat{x}_{\mu_{2}} \ldots \hat{x}_{\mu_{n}}$ and $D_{\nu_{1}} D_{\nu_{2}} \ldots D_{\nu_{m}}$, is said to be $S O_{a}(n)$-invariant if

$$
\left[M_{\mu \nu}, f(\hat{x}, D)\right]=0 \quad \text { for all } \quad \mu, \nu
$$

We introduce the $S O_{a}(n)$-invariant Laplace operator $\square$ satisfying the commutation relations

$$
\begin{aligned}
{\left[M_{\mu \nu}, \square\right] } & =0, \\
{\left[\square, \hat{x}_{\mu}\right] } & =2 D_{\mu} .
\end{aligned}
$$

The Laplace operator can be expressed in terms of the operator $D^{2}$. Let us assume the Ansatz $\square=F\left(D^{2}\right)$, where $F$ is analytic, and impose the boundary condition $F\left(D^{2}\right) \rightarrow \partial^{2}$ as $a \rightarrow 0$. Then Eq. (11) is automatically satisfied, and Eqs. (9) and (12) together with the boundary condition yield

$$
\square=\frac{2}{a^{2}}\left(1-\sqrt{1-a^{2} D^{2}}\right) .
$$


It follows from here that

$$
D^{2}=\square\left(1-\frac{a^{2}}{4} \square\right)
$$

\subsection{The shift operator}

At this point it is convenient to introduce the shift operator $Z$ via the commutation relations

$$
\begin{aligned}
{\left[Z, \hat{x}_{\mu}\right] } & =i a_{\mu} Z \\
{\left[Z, D_{\mu}\right] } & =0 .
\end{aligned}
$$

We assume that $Z \rightarrow 1$ in the limit as $a \rightarrow 0$. The shift operator acts on an arbitrary function $f(\hat{x})$ by

$$
Z f(\hat{x})=f(\hat{x}+i a) Z
$$

We note that the inverse shift operator $Z^{-1}$ satisfies the same relations as $Z$ when $a_{\mu}$ is replaced by $-a_{\mu}$. The shift operator can be expressed in terms of the Dirac derivative $D$ as follows. Using Eqs. (9) and (12) one can verify that

$$
\left[-i a D-\frac{a^{2}}{2} \square, \hat{x}_{\mu}\right]=-i a_{\mu}\left(-i a D+\sqrt{1-a^{2} D^{2}}\right)
$$

where $a D=a_{\mu} D_{\mu}$. Inserting Eq. (13) for the Laplace operator into Eq. (18), we obtain $\left[Z^{-1}, \hat{x}_{\mu}\right]=-i a_{\mu} Z^{-1}$, where

$$
Z^{-1}=-i a D+\sqrt{1-a^{2} D^{2}} .
$$

Inverting the above expression, we find

$$
Z=\frac{i a D+\sqrt{1-a^{2} D^{2}}}{1-a^{2} D^{2}+(a D)^{2}} .
$$

It is interesting to note that the algebra generated by $\hat{x}_{\mu}, M_{\mu \nu}$ and $D_{\mu}$ can be described using the shift operator $Z$ as

$$
\begin{aligned}
\hat{x}_{\mu} Z \hat{x}_{\nu} & =x_{\nu} Z \hat{x}_{\mu}, \\
{\left[D_{\mu}, \hat{x}_{\nu}\right] } & =\delta_{\mu \nu} Z^{-1}+i a_{\mu} D_{\nu} .
\end{aligned}
$$


The remaining commutation relations for $\left[M_{\mu \nu}, M_{\lambda \rho}\right],\left[M_{\mu \nu}, \hat{x}_{\lambda}\right]$ and $\left[M_{\mu \nu}, D_{\lambda}\right]$ are satisfied by representing $M_{\mu \nu}$ in a unique way as

$$
M_{\mu \nu}=\left(\hat{x}_{\mu} D_{\nu}-\hat{x}_{\nu} D_{\mu}\right) Z
$$

We will justify this relation later when we consider the so-called natural realization in Sect. 3,

\subsection{The Leibniz rule and coproduct}

Now we turn our attention to the generalized Leibniz rule and coproduct. The commutator of $M_{\mu \nu}$ with an arbitrary function $f(\hat{x})$ is given by

$$
\begin{aligned}
{\left[M_{\mu \nu}, f\right] } & =\left(M_{\mu \nu} f\right) \\
& +i a_{\mu}\left[\left(D_{\lambda}-\frac{i a_{\lambda}}{2} \square\right) Z f\right] M_{\lambda \nu}-i a_{\nu}\left[\left(D_{\lambda}-\frac{i a_{\lambda}}{2} \square\right) Z f\right] M_{\lambda \mu} .
\end{aligned}
$$

This relation can be shown by using Eq. (6) and proceeding by induction on the degree of monomials in $\hat{x}_{\mu}$. From Eq. (24) we obtain the coproduct for $M_{\mu \nu}$,

$$
\begin{aligned}
\triangle M_{\mu \nu} & =M_{\mu \nu} \otimes \mathbf{1}+\mathbf{1} \otimes M_{\mu \nu} \\
& +i a_{\mu}\left(D_{\lambda}-\frac{i a_{\lambda}}{2} \square\right) Z \otimes M_{\lambda \nu}-i a_{\nu}\left(D_{\lambda}-\frac{i a_{\lambda}}{2} \square\right) Z \otimes M_{\lambda \mu}
\end{aligned}
$$

Similarly, one can show that the commutator of $D_{\mu}$ with $f(\hat{x})$ is given by

$$
\left[D_{\mu}, f\right]=\left(D_{\mu} f\right) Z^{-1}+i a_{\mu}\left(D_{\lambda} Z f\right) D_{\lambda}-\frac{i a_{\mu}}{2}(\square Z f) i a D
$$

hence for the coproduct we have

$$
\triangle D_{\mu}=D_{\mu} \otimes Z^{-1}+\mathbf{1} \otimes D_{\mu}+i a_{\mu}\left(D_{\lambda} Z\right) \otimes D_{\lambda}-\frac{i a_{\mu}}{2} \square Z \otimes i a D
$$

Furthermore, the coproduct for the shift operator $Z$ is simply

$$
\triangle Z=Z \otimes Z
$$

Some examples of the Poincaré invariant interpretation of $\mathrm{NC}$ spaces and of the twisted Poincaré coalgebra were also considered in [38]-[40]. 
It is interesting to note that the operators $D_{\mu}, \square$ and $Z$ can be expressed in terms of auxiliary derivatives $\partial_{\mu}^{L}$ and $\partial_{\mu}^{R}$ satisfying the following commutation relations:

$$
\begin{aligned}
& {\left[\partial_{\mu}^{L}, \partial_{\nu}^{L}\right]=0,} \\
& {\left[\partial_{\mu}^{L}, \hat{x}_{\nu}\right]=\delta_{\mu \nu} Z^{-1},}
\end{aligned}
$$

and

$$
\begin{aligned}
{\left[\partial_{\mu}^{R}, \partial_{\nu}^{R}\right] } & =0, \\
{\left[\partial_{\mu}^{R}, \hat{x}_{\nu}\right] } & =\delta_{\mu \nu}+i a_{\nu} \partial_{\mu}^{R} .
\end{aligned}
$$

Equations (29)-(30) and (31)-(32) are consistent with the commutation relation (11), and hence both sets of equations define a deformed Heisenberg algebra. One can think of $\partial_{\mu}^{L}$ and $\partial_{\mu}^{R}$ as "left" and "right" deformations of the ordinary derivative $\partial_{\mu}$. Indeed, using the commutation relations (30) and (32) one can show that the coproducts of $\partial_{\mu}^{L}$ and $\partial_{\mu}^{R}$ are given by

$$
\begin{aligned}
& \triangle \partial_{\mu}^{L}=\partial_{\mu}^{L} \otimes Z^{-1}+\mathbf{1} \otimes \partial_{\mu}^{L}, \\
& \triangle \partial_{\mu}^{R}=\partial_{\mu}^{R} \otimes \mathbf{1}+Z \otimes \partial_{\mu}^{R},
\end{aligned}
$$

and $\partial_{\mu}^{R}=\partial_{\mu}^{L} Z$. Hence, the coproducts $\triangle \partial_{\mu}^{L}$ and $\triangle \partial_{\mu}^{R}$ are left and right deformations of the ordinary coproduct $\triangle \partial_{\mu}=\partial_{\mu} \otimes \mathbf{1}+\mathbf{1} \otimes \partial_{\mu}$, respectively. In fact, the coproducts $\triangle \partial_{\mu}^{L}$ and $\triangle \partial_{\mu}^{R}$ are given by Eqs. (33)-(134) if and only if Eqs. (30) and (32) hold. One can show that the operators $D_{\mu}, \square$ and $Z$ are expressed in terms of the left and right deformation derivatives as

$$
\begin{aligned}
D_{\mu} & =\partial_{\mu}^{L}+\frac{i a_{\mu}}{2} \square \\
\square & =\left(\partial^{L}\right)^{2} Z \\
Z & =1+i\left(a \partial^{L}\right) Z=\frac{1}{1-i a \partial^{L}}
\end{aligned}
$$

and

$$
\begin{aligned}
D_{\mu} & =\partial_{\mu}^{R} Z^{-1}+\frac{i a_{\mu}}{2} \square, \\
\square & =\left(\partial^{R}\right)^{2} Z^{-1}, \\
Z & =1+i a \partial^{R} .
\end{aligned}
$$


The algebra generated by $\hat{x}_{\mu}, M_{\mu \nu}$ and $D_{\mu}$ is covariant under the action of the rotation group $S O(n)$. Indeed, let $R \in S O(n)$ be a rotation matrix and denote the transformed variables by $\hat{x}_{\mu}^{\prime}=R_{\mu \alpha} \hat{x}_{\alpha}, D_{\mu}^{\prime}=R_{\mu \alpha} D_{\alpha}, M_{\mu \nu}^{\prime}=R_{\mu \alpha} R_{\nu \beta} M_{\alpha \beta}$ and $a_{\mu}^{\prime}=R_{\mu \alpha} a_{\alpha}$. Then Eqs. (13) and (20) immediately yield

$$
\square^{\prime}=\square \quad \text { and } \quad Z^{\prime}=Z,
$$

and the transformed generators $\hat{x}_{\mu}^{\prime}, M_{\mu \nu}^{\prime}$ and $D_{\mu}^{\prime}$ satisfy the relations

$$
\begin{aligned}
\hat{x}_{\mu}^{\prime} Z \hat{x}_{\nu}^{\prime} & =\hat{x}_{\nu}^{\prime} Z \hat{x}_{\mu}^{\prime}, \\
{\left[D_{\mu}^{\prime}, \hat{x}_{\nu}^{\prime}\right] } & =\delta_{\mu \nu} Z^{-1}+i a_{\mu}^{\prime} D_{\nu}^{\prime}, \\
M_{\mu \nu}^{\prime} & =\left(\hat{x}_{\mu}^{\prime} D_{\nu}^{\prime}-\hat{x}_{\nu}^{\prime} D_{\mu}^{\prime}\right) Z .
\end{aligned}
$$

\section{Covariant realizations}

A realization of the $\mathrm{NC}$ coordinates $\hat{x}_{\mu}$ in terms of ordinary commutative coordinates and their derivatives was found using the Bargman representation and the methods developed in [29], 30], 334, [35]. The goal of this section is to find covariant realizations of the algebra generated by the NC coordinates $\hat{x}_{\mu}$, the rotation generators $M_{\mu \nu}$ and the Dirac derivatives $D_{\mu}$. The realizations are found in terms of functions of ordinary coordinates $x_{1}, x_{2}, \ldots, x_{n}$ and their derivatives $\partial_{1}, \partial_{2}, \ldots, \partial_{n}$ which generate the Heisenberg algebra $\left[x_{\mu}, x_{\nu}\right]=\left[\partial_{\mu}, \partial_{\nu}\right]=0$ and $\left[\partial_{\mu}, x_{\nu}\right]=\delta_{\mu \nu}$. In general, these functions will satisfy a system of coupled partial differential equations (PDE) determined by the commutation relations for $\hat{x}_{\mu}, M_{\mu \nu}$ and $D_{\mu}$. In the following we derive such systems of PDE's and then we consider their solutions.

The most general Ansatz for $\hat{x}_{\mu}$ is

$$
\hat{x}_{\mu}=x_{\alpha} \Phi_{\alpha \mu}(A, B),
$$

where $\Phi_{\alpha \mu}$ is a function of the commuting variables $A=i a \partial$ and $B=a^{2} \partial^{2}$, and satisfies the boundary condition $\Phi_{\alpha \mu}(0,0)=\delta_{\alpha \mu}$. This realization is covariant under the orthogonal transformation $R \in S O(n)$ (c.f. Sect. 2.2 and $x_{\mu}^{\prime}=R_{\mu \alpha} x_{\alpha}, \partial_{\nu}^{\prime}=$ $\left.R_{\mu \alpha} \partial_{\alpha}\right)$, i.e. under the action of the generators

$$
M_{\mu \nu}^{0}=x_{\mu} \partial_{\nu}-x_{\nu} \partial_{\mu}+a_{\mu} \frac{\partial}{\partial a_{\nu}}-a_{\nu} \frac{\partial}{\partial a_{\mu}} .
$$


We consider a particular form of the above Ansatz given by

$$
\hat{x}_{\mu}=x_{\mu} \varphi+i(a x)\left(\partial_{\mu} \beta_{1}+i a_{\mu} \partial^{2} \beta_{2}\right)+i(x \partial)\left(a_{\mu} \gamma_{1}+i a^{2} \partial_{\mu} \gamma_{2}\right)
$$

where $\varphi, \beta_{i}$ and $\gamma_{i}$ are functions of $A$ and $B$. We impose the boundary conditions $\varphi(0,0)=1$ and $\beta_{i}(0,0), \gamma_{i}(0,0)$ finite in order to ensure the smooth limit $\hat{x}_{\mu} \rightarrow x_{\mu}$ as $a \rightarrow 0$. Substituting the Ansatz (47) into Eq. (1), we obtain the following system of equations:

$$
\begin{gathered}
\frac{\partial \varphi}{\partial A} \varphi-B\left(\frac{\partial \varphi}{\partial A}-2 A \frac{\partial \varphi}{\partial B}\right) \beta_{2}+\left(A \frac{\partial \varphi}{\partial A}+2 B \frac{\partial \varphi}{\partial B}\right) \gamma_{1}-\varphi\left(\gamma_{1}-1\right)=0 \\
2 \frac{\partial \varphi}{\partial B} \varphi-\left(\frac{\partial \varphi}{\partial A}-2 A \frac{\partial \varphi}{\partial B}\right) \beta_{1}-\left(A \frac{\partial \varphi}{\partial A}+2 B \frac{\partial \varphi}{\partial B}\right) \gamma_{2}+\varphi \gamma_{2}=0 \\
\left(\frac{\partial \beta_{1}}{\partial A}-2 B \frac{\partial \beta_{2}}{\partial B}\right) \varphi-B\left(\frac{\partial \beta_{1}}{\partial A}-2 A \frac{\partial \beta_{1}}{\partial B}\right) \beta_{2}+B\left(\frac{\partial \beta_{2}}{\partial A}-2 A \frac{\partial \beta_{2}}{\partial B}\right) \beta_{1} \\
+\left(A \frac{\partial \beta_{1}}{\partial A}+2 B \frac{\partial \beta_{2}}{\partial B}\right) \gamma_{1}+B\left(A \frac{\partial \beta_{2}}{\partial A}+2 B \frac{\partial \beta_{2}}{\partial B}\right) \gamma_{2} \\
-\left(\beta_{1}^{2}+2 A \beta_{1} \beta_{2}\right)+B \beta_{2} \gamma_{2}-2 \beta_{2} \varphi+\beta_{1}=0 \\
+\left(A \frac{\partial \gamma_{1}}{\partial B}+\frac{\partial \gamma_{2}}{\partial A}\right) \varphi+\left(\frac{\partial \gamma_{1}}{\partial A}-2 A \frac{\partial \gamma_{1}}{\partial B}\right) \beta_{1}+\left(\frac{\partial \gamma_{2}}{\partial A}-2 A \frac{\partial \gamma_{2}}{\partial B}\right) B \beta_{2} \\
+\left(A \frac{\partial \gamma_{1}}{\partial B}\right) \gamma_{2}-\left(A \frac{\partial \gamma_{2}}{\partial A}+2 B \frac{\partial \gamma_{2}}{\partial B}\right) \gamma_{1}+\gamma_{2}\left(\beta_{1}-\gamma_{1}-1\right)=0
\end{gathered}
$$

Next, we consider realizations of the rotation algebra $S O_{a}(n)$. We assume that the rotation generators are given by the Ansatz

$$
M_{\mu \nu}=\left(x_{\mu} \partial_{\nu}-x_{\nu} \partial_{\mu}\right) \mathcal{F}_{1}+i(x \partial)\left(a_{\mu} \partial_{\nu}-a_{\nu} \partial_{\mu}\right) \mathcal{F}_{2}+i\left(x_{\mu} a_{\nu}-x_{\nu} a_{\mu}\right) \partial^{2} \mathcal{F}_{3},
$$

where the functions $\mathcal{F}_{1}, \mathcal{F}_{2}$ and $\mathcal{F}_{3}$ depend on $A$ and $B$, and satisfy the boundary conditions $\mathcal{F}_{1}(0,0)=1$ and $F_{2}(0,0), \mathcal{F}_{3}(0,0)$ finite, respectively. The boundary conditions imply that $M_{\mu \nu}$ becomes the ordinary rotation generator as $a \rightarrow 0$. The Ansatz is antisymmetric in the indices $\mu$ and $\nu$. Now we can calculate the commutator $\left[M_{\mu \nu}, M_{\lambda \rho}\right]$ by inserting the Ansatz (52) into Eq. (44). This results in the system of equations

$$
\mathcal{F}_{1}=1, \quad \frac{\partial \mathcal{F}_{3}}{\partial A}+\left(\mathcal{F}_{3}+A \frac{\partial \mathcal{F}_{3}}{\partial A}\right) \mathcal{F}_{2}-2 \mathcal{F}_{3}^{2}=0
$$

Since $\mathcal{F}_{2}$ is uniquely determined by $\mathcal{F}_{3}$, these equations provide a realization of the algebra $S O_{a}(n)$ in terms of an arbitrary parameter function $\mathcal{F}_{3}$. However, $M_{\mu \nu}$ and 
$\hat{x}_{\mu}$ are required to form the extended Lie algebra (6), hence $\mathcal{F}_{3}$ is related with the functions $\varphi, \beta_{i}$ and $\gamma_{i}$. This relation can be shown either directly from Eq. (6) or by using a realization of the Dirac operator $D_{\mu}$ and Eq. (23).

We assume that the Dirac operator is given by

$$
D_{\mu}=\partial_{\mu} \mathcal{G}_{1}+i a_{\mu} \partial^{2} \mathcal{G}_{2}
$$

Here the functions $\mathcal{G}_{1}$ and $\mathcal{G}_{2}$ depend on $A$ and $B$, and satisfy the boundary conditions $\mathcal{G}_{1}(0,0)=1$ and $\mathcal{G}_{2}(0,0)$ finite, respectively. Inserting Eq. (54) into Eq. (9), we obtain

$$
\begin{gathered}
\sqrt{1-a^{2} D^{2}}-i a D=\mathcal{G}_{1} \varphi \\
\frac{\partial \mathcal{G}_{1}}{\partial A} \varphi+\left(-\frac{\partial \mathcal{G}_{1}}{\partial A}+2 A \frac{\partial \mathcal{G}_{1}}{\partial B}\right) B \beta_{2}+\left(\mathcal{G}_{1}+A \frac{\partial \mathcal{G}_{1}}{\partial A}+2 B \frac{\partial \mathcal{G}_{1}}{\partial B}\right) \gamma_{1}=0 \\
2 \frac{\partial \mathcal{G}_{1}}{\partial B} \varphi+\left(2 A \frac{\partial \mathcal{G}_{1}}{\partial B}-\frac{\partial \mathcal{G}_{1}}{\partial A}\right) \beta_{1}-\left(\mathcal{G}_{1}+A \frac{\partial \mathcal{G}_{1}}{\partial A}+2 B \frac{\partial \mathcal{G}_{1}}{\partial B}\right) \gamma_{2}=0 \\
\mathcal{G}_{1} \beta_{2}+\frac{\partial \mathcal{G}_{2}}{\partial A} \varphi+2 A \mathcal{G}_{2} \beta_{2}+B\left(2 A \frac{\partial \mathcal{G}_{2}}{\partial B}-\frac{\partial \mathcal{G}_{2}}{\partial A}\right) \beta_{2} \\
+\left(2 \mathcal{G}_{2}+A \frac{\partial \mathcal{G}_{2}}{\partial A}+2 B \frac{\partial \mathcal{G}_{2}}{\partial B}\right) \gamma_{1}=\mathcal{G}_{2} \\
\mathcal{G}_{1} \beta_{1}+\left(2 \mathcal{G}_{2}+2 B \frac{\partial \mathcal{G}_{2}}{\partial B}\right) \varphi+2 A \mathcal{G}_{2} \beta_{1}+\left(2 A \frac{\partial \mathcal{G}_{2}}{\partial B}-\frac{\partial \mathcal{G}_{2}}{\partial A}\right) B \beta_{1} \\
-\left(2 \mathcal{G}_{2}+\frac{\partial \mathcal{G}_{2}}{\partial A} A+2 B \frac{\partial \mathcal{G}_{2}}{\partial B}\right) B \gamma_{2}=\mathcal{G}_{1}
\end{gathered}
$$

Equations (55) and (20) imply that the inverse shift operator has a simple realization as

$$
Z^{-1}=\mathcal{G}_{1} \varphi
$$

The system of PDE's for the unknown functions $\varphi, \beta_{i}, \gamma_{i}, \mathcal{F}_{i}$ and $\mathcal{G}_{i}$ is too difficult to solve in full generality. We will reduce the system to a manageable form by considering special choices for the functions $\beta_{1}$ and $\beta_{2}: \beta_{1}=\beta_{2}=0$ and $\beta_{1}=1$, $\beta_{2}=0$. In each case, we obtain an infinite family of realizations parametrized by the function $\varphi$. We call these realizations type I and type II, respectively. 
Realization I: $\beta_{1}=\beta_{2}=0$.

In the first realization, Eqs. (47)-(51) imply

$$
\hat{x}_{\mu}=x_{\mu} \varphi+i(x \partial)\left(a_{\mu} \gamma_{1}+i a^{2} \partial_{\mu} \gamma_{2}\right)
$$

where

$$
\begin{aligned}
\gamma_{1} & =\frac{\left(1+\frac{\partial \varphi}{\partial A}\right) \varphi}{\varphi-\left(A \frac{\partial \varphi}{\partial A}+2 B \frac{\partial \varphi}{\partial B}\right)} \\
\gamma_{2} & =-\frac{2 \frac{\partial \varphi}{\partial B} \varphi}{\varphi-\left(A \frac{\partial \varphi}{\partial A}+2 B \frac{\partial \varphi}{\partial B}\right)}
\end{aligned}
$$

Furthermore, Eqs. (156)-(159) yield

$$
\mathcal{G}_{1}=\frac{1}{\varphi+A}, \quad \mathcal{G}_{2}=\frac{1}{2 \varphi(\varphi+A)} .
$$

We note that in view of Eq. (60) the shift operator is given by

$$
Z=1+\frac{A}{\varphi}
$$

We are now able to find realizations of the rotation generators $M_{\mu \nu}$. Inserting the realizations for $\hat{x}_{\mu}, D_{\mu}$ and $Z$ into Eq. (23) and comparing the obtained expression with Eq. (52), we find

$$
\mathcal{F}_{1}=1, \quad \mathcal{F}_{2}=\frac{\gamma_{1}}{\varphi}+\frac{B \gamma_{2}}{2 \varphi^{2}}=\frac{\left(1+\frac{\partial \varphi}{\partial A}\right) \varphi-B \frac{\partial \varphi}{\partial B}}{\varphi^{2}-\left(A \frac{\partial \varphi}{\partial A}+2 B \frac{\partial \varphi}{\partial B}\right) \varphi}, \quad \mathcal{F}_{3}=\frac{1}{2 \varphi}
$$

Note that $\mathcal{F}_{1}=1$ is consistent with the earlier obtained expression in Eq. (53). Next, we find a realization of the Laplace operator $\square$ which is uniquely determined by Eq. (13). Using the realizations for $Z$ and $D_{\mu}$ in Eqs. (64) and (65) we obtain $\sqrt{1-a^{2} D^{2}}=1-a^{2} \partial^{2} \mathcal{G}_{2}$. Therefore, Eq. (13) yields

$$
\square=\partial^{2} \mathcal{H}, \quad \text { where } \quad \mathcal{H}=\frac{1}{\varphi(\varphi+A)} .
$$

Note that since $\varphi(0,0)=1$ and $A \rightarrow 0$ as $a \rightarrow 0$, in the limiting case we have $\square \rightarrow \partial^{2}$ as $a \rightarrow 0$. 
Realization II: $\beta_{1}=1, \beta_{2}=0$.

Repeating the above calculations in the second realization we find

$$
\hat{x}_{\mu}=x_{\mu} \varphi+i(a x) \partial_{\mu}+i(a \partial)\left(a_{\mu} \gamma_{1}+i a^{2} \partial_{\mu} \gamma_{2}\right)
$$

where

$$
\begin{aligned}
\gamma_{1} & =\frac{\left(1+\frac{\partial \varphi}{\partial A}\right) \varphi}{\varphi-\left(A \frac{\partial \varphi}{\partial A}+2 B \frac{\partial \varphi}{\partial B}\right)}, \\
\gamma_{2} & =\frac{\frac{\partial \varphi}{\partial A}-2(\varphi+A) \frac{\partial \varphi}{\partial B}}{\varphi-\left(A \frac{\partial \varphi}{\partial A}+2 B \frac{\partial \varphi}{\partial B}\right)} .
\end{aligned}
$$

The functions $\mathcal{G}_{1}$ and $\mathcal{G}_{2}$ are given by

$$
\mathcal{G}_{1}=\frac{1}{\sqrt{(\varphi+A)^{2}+B}}, \quad \mathcal{G}_{2}=0
$$

and the shift operator yields

$$
Z=\sqrt{\left(1+\frac{A}{\varphi}\right)^{2}+\frac{B}{\varphi^{2}}}
$$

Similarly, for $\mathcal{F}_{1}, \mathcal{F}_{2}$ and $\mathcal{F}_{3}$ we obtain

$$
\mathcal{F}_{1}=1, \quad \mathcal{F}_{2}=\frac{\gamma_{1}}{\varphi}=\frac{1+\frac{\partial \varphi}{\partial A}}{\varphi-\left(A \frac{\partial \varphi}{\partial A}+2 B \frac{\partial \varphi}{\partial B}\right)}, \quad \mathcal{F}_{3}=0 .
$$

Note that $\mathcal{F}_{1}, \mathcal{F}_{2}$ and $\mathcal{F}_{3}$ in all realizations are consistent with Eq. (23). Furthermore, for the Laplace operator we have $\square=\partial^{2} \mathcal{H}$ where

$$
\mathcal{H}=\frac{2}{B}\left(1-(\varphi+A) \mathcal{G}_{1}\right)=\frac{2}{B}\left(1-\frac{\varphi+A}{\sqrt{(\varphi+A)^{2}+B}}\right) .
$$

\subsection{Special realizations}

Of particular interest are some realizations obtained for a special choice of the parameter function $\varphi$. In the realization of type I we consider the left, right and symmetric realizations corresponding to $\varphi_{L}=1-A, \varphi_{R}=1$ and $\varphi_{S}=A /(\exp (A)-1)$, respectively. One can show that in the left realization the derivative operator $\partial_{\mu}$ becomes the left deformation derivative $\partial_{\mu}^{L}$. Similarly, in the right realization the derivative 
operator $\partial_{\mu}$ becomes the right deformation derivative $\partial_{\mu}^{R}$. The symmetric realization is related to Weyl's symmetric ordering of the monomials in $\hat{x}_{\mu}$. In the realization of type II we consider the natural realization corresponding to $\varphi_{N}=-A+\sqrt{1-B}$. In this realization the Dirac derivative is simply given by $D_{\mu}=\partial_{\mu}$.

Left realization: $\beta_{1}=\beta_{2}=0, \varphi_{L}=1-A$.

Inserting $\varphi_{L}=1-A$ into Eqs. (61) -(67), we find

$$
\begin{aligned}
\hat{x}_{\mu} & =x_{\mu}(1-A), \\
M_{\mu \nu} & =x_{\mu} \partial_{\nu}-x_{\nu} \partial_{\mu}+\frac{1}{2} i\left(x_{\mu} a_{\nu}-x_{\nu} a_{\mu}\right) \frac{1}{1-A} \partial^{2}, \\
D_{\mu} & =\partial_{\mu}+\frac{i a_{\mu}}{2} \square, \\
Z & =\frac{1}{1-A}, \\
\square & =\frac{1}{1-A} \partial^{2} .
\end{aligned}
$$

It follows form Eqs. (75) and (78) that

$$
\left[\partial_{\mu}, \hat{x}_{\nu}\right]=\delta_{\mu \nu} Z^{-1}
$$

Thus, in view of Eq. (30), we see that $\partial_{\mu}$ is the left deformation derivative $\partial_{\mu}^{L}$.

Right realization: $\beta_{1}=\beta_{2}=0, \varphi_{R}=1$.

Repeating the calculations with $\varphi_{R}=1$, we obtain

$$
\begin{aligned}
\hat{x}_{\mu} & =x_{\mu}+i a_{\mu}(x \partial), \\
M_{\mu \nu} & =x_{\mu} \partial_{\nu}-x_{\nu} \partial_{\mu}+i(x \partial)\left(a_{\mu} \partial_{\nu}-a_{\nu} \partial_{\mu}\right)+\frac{i}{2}\left(x_{\mu} a_{\nu}-a_{\nu} x_{\mu}\right) \partial^{2}, \\
D_{\mu} & =\frac{1}{1+A} \partial_{\mu}+\frac{i a_{\mu}}{2} \square, \\
Z & =1+A \\
\square & =\frac{1}{1+A} \partial^{2} .
\end{aligned}
$$

In this case,

$$
\left[\partial_{\mu}, \hat{x}_{\nu}\right]=\delta_{\mu \nu}+i a_{\nu} \partial_{\mu}
$$


hence a comparison with Eq. (32) shows that $\partial_{\mu}$ is the right deformation derivative $\partial_{\mu}^{R}$.

Symmetric realization: $\beta_{1}=\beta_{2}=0, \varphi_{S}=A /(\exp (A)-1)$.

This realization corresponds to the symmetric Weyl ordering [22]. It also follows from the universal formula for a general Lie algebra [41], after inserting the structure constants from Eq. (2). We have

$$
\begin{aligned}
\hat{x}_{\mu} & =x_{\mu} \frac{A}{e^{A}-1}+i a_{\mu}(x \partial) \frac{e^{A}-1-A}{\left(e^{A}-1\right) A}, \\
M_{\mu \nu} & =x_{\mu} \partial_{\nu}-x_{\nu} \partial_{\mu}+i(x \partial)\left(a_{\mu} \partial_{\nu}-a_{\nu} \partial_{\mu}\right) \frac{e^{A}-1-A}{A^{2}}, \\
D_{\mu} & =\frac{e^{A}-1}{A e^{A}} \partial_{\mu}+\frac{i a_{\mu}}{2} \square, \\
Z & =e^{A} \\
\square & =\frac{\left(e^{A}-1\right)^{2}}{A^{2} e^{A}} \partial^{2}
\end{aligned}
$$

and

$$
\left[\partial_{\mu}, \hat{x}_{\nu}\right]=\delta_{\mu \nu} \varphi_{S}+i a_{\nu} \partial_{\mu} \frac{1-\varphi_{S}}{A} .
$$

Natural realization: $\beta_{1}=1, \beta_{2}=0, \varphi_{N}=-A+\sqrt{1-B}$.

The natural realization is a special case of the realization of type II in which the Dirac derivative $D_{\mu}=\partial_{\mu} \mathcal{G}_{1}+i a_{\mu} \partial^{2} \mathcal{G}_{2}$ simplifies to $D_{\mu}=\partial_{\mu}$. In view of Eq. (71), this holds when $\varphi_{N}=-A+\sqrt{1-B}$. Then we have

$$
\begin{aligned}
\hat{x}_{\mu} & =x_{\mu}(-A+\sqrt{1-B})+i(a x) \partial_{\mu}, \\
M_{\mu \nu} & =x_{\mu} \partial_{\nu}-x_{\nu} \partial_{\mu}, \\
D_{\mu} & =\partial_{\mu}, \\
Z & =\frac{1}{-A+\sqrt{1-B}}, \\
\square & =\frac{2}{B}(1-\sqrt{1-B}) \partial^{2} .
\end{aligned}
$$

In the natural realization it is easily shown that the rotation generators $M_{\mu \nu}$ are given by Eq. (23). Indeed, Eqs. (93) and (95) imply that

$$
\left(\hat{x}_{\mu} D_{\nu}-\hat{x}_{\nu} D_{\mu}\right) Z=\left(x_{\mu} \partial_{\nu}-x_{\nu} \partial_{\mu}\right) \varphi Z \text {. }
$$


However, $\varphi Z=1$, thus Eqs. (94) and (98) yield

$$
M_{\mu \nu}=\left(\hat{x}_{\mu} D_{\nu}-\hat{x}_{\nu} D_{\mu}\right) Z
$$

\subsection{Hermiticity}

All relations of the type $\left[\hat{x}_{\mu}, \hat{x}_{\nu}\right],\left[M_{\mu \nu}, M_{\lambda \rho}\right],\left[M_{\mu \nu}, \hat{x}_{\lambda}\right],\left[D_{\mu}, D_{\nu}\right],\left[M_{\mu \nu}, D_{\lambda}\right],\left[D_{\mu}, \hat{x}_{\nu}\right]$, i.e. Eqs. (1), (4), (6), (7), (8) and (9), are invariant under the formal antilinear involution:

$$
\hat{x}_{\mu}^{\dagger}=\hat{x}_{\mu}, \quad D_{\mu}^{\dagger}=-D_{\mu}, \quad M_{\mu \nu}^{\dagger}=-M_{\mu \nu}, \quad c^{\dagger}=\bar{c}, \quad c \in \mathbb{C} .
$$

The order of elements in the product is inverted under the involution. The commutative coordinates $x_{\mu}$ and their derivatives $\partial_{\mu}$ also satisfy the involution property: $x_{\mu}^{\dagger}=x_{\mu}$ and $\partial_{\mu}^{\dagger}=-\partial_{\mu}$. Then the NC coordinates $\hat{x}_{\mu}$ are represented by hermitian operators. However, Eq. (45) is generally not hermitian. The hermitian representations are simply obtained by the following expression [22]:

$$
\hat{x}_{\mu}^{h}=\frac{1}{2}\left(x_{\alpha} \Phi_{\alpha \mu}+\left(\Phi^{\dagger}\right)_{\mu \alpha} x_{\alpha}\right) .
$$

However, the physical results do not depend on the choice of representation as long as there exists a smooth limit $\hat{x}_{\mu} \rightarrow x_{\mu}$ as $a \rightarrow 0$. Therefore, we restrict ourselves to non-hermitian realizations only.

\section{Star product}

Recall that in Sect. 3 a general Ansatz for the NC coordinates was introduced,

$$
\hat{x}_{\mu}=x_{\alpha} \Phi_{\alpha \mu}(A, B), \quad \Phi_{\alpha \mu}(0,0)=\delta_{\alpha \mu} .
$$

Let us define the vacuum state by $|0\rangle=1$ and $\partial_{\mu}|0\rangle=0$, and fix the normalization condition by $\hat{x}_{\mu}|0\rangle=x_{\mu}$. For a given realization $\Phi_{\mu \nu}$ there is a unique map sending mononials in the $\mathrm{NC}$ coordinates $\hat{x}_{\mu}$ into polynomials of the commutative coordinates $x_{\mu}$. This map is given by

$$
\prod_{i=1}^{k} \hat{x}_{\mu_{i}}|0\rangle=P_{k}(x)
$$


where $P_{k}$ is a polynomial of degree $k$. We also have the dual relation

$$
\prod_{i=1}^{k} x_{\mu_{i}}=\hat{P}_{k}(\hat{x})|0\rangle
$$

where $\hat{P}_{k}$ is also a polynomial of degree $k$ in $\hat{x}$. For example, in the left realization we have

$$
\prod_{i=1}^{k} \hat{x}_{\mu_{i}}|0\rangle=x_{\mu_{1}}\left(x_{\mu_{2}}-i a_{\mu_{2}}\right)\left(x_{\mu_{3}}-i 2 a_{\mu_{3}}\right) \ldots\left(x_{\mu_{k}}-i(k-1) a_{\mu_{k}}\right),
$$

together with the dual relation

$$
\prod_{i=1}^{k} x_{\mu_{i}}=\hat{x}_{\mu_{1}} Z \hat{x}_{\mu_{2}} \ldots Z \hat{x}_{\mu_{k}}|0\rangle \text {. }
$$

Similarly, in the right realization we find

$$
\begin{aligned}
\prod_{i=1}^{k} \hat{x}_{\mu_{i}}|0\rangle & =\left(x_{\mu_{1}}+i(k-1) a_{\mu_{1}}\right)\left(x_{\mu_{2}}+i(k-2) a_{\mu_{2}}\right) \ldots\left(x_{\mu_{k-1}}+i a_{\mu_{k-1}}\right) x_{\mu_{k}}, \\
\prod_{i=1}^{k} x_{\mu_{i}} & =Z^{-(k-1)} \hat{x}_{\mu_{1}} Z \hat{x}_{\mu_{2}} \ldots Z \hat{x}_{\mu_{k}}|0\rangle .
\end{aligned}
$$

One can obtain similar expressions for the symmetric realization [22]. It is interesting to note that in the realization of type I when $\varphi=\varphi(A)$ the following relation holds:

$$
e^{i k \hat{x}}|0\rangle=\exp \left(i \frac{\varphi(-a k)}{\varphi_{S}(-a k)} k x\right)
$$

where $k \in \mathbb{R}^{n}$ and $\varphi_{S}(A)=A /(\exp (A)-1)$. In particular, in the symmetric realization when $\varphi=\varphi_{S}$ we have

$$
e^{i k \hat{x}}|0\rangle=e^{i k x}
$$

Similarly, in the natural realization one can show that

$$
e^{i k \hat{x}}|0\rangle=e^{i P_{N}(k) x}
$$

where

$$
P_{N}(k)_{\mu}=\frac{1}{\varphi_{S}(a k)}\left(k_{\mu}-\frac{k^{2}}{2 \varphi_{S}(-a k)} a_{\mu}\right) .
$$


Equation (103) defines an isomorphism of the universal enveloping algebras generated by $\hat{x}_{1}, \hat{x}_{2}, \ldots, \hat{x}_{n}$ and $x_{1}, x_{2}, \ldots, x_{n}$, respectively. This can be extended to formal power series by

$$
\hat{f}(\hat{x})|0\rangle=f(x)
$$

where the function $f$ depends on the realization $\Phi_{\mu \nu}$. The Leibniz rule and the related coproduct $\triangle \partial_{\mu}$ follow uniquely from the commutator relation

$$
\left[\partial_{\mu}, \hat{x}_{\nu}\right]=\Phi_{\mu \nu}(A, B)
$$

and the conditions $\partial_{\mu}|0\rangle=0, \hat{x}_{\mu}|0\rangle=x_{\mu}$.

The star product of two functions $f(x)$ and $g(x)$ is defined by

$$
(f \star g)(x)=\hat{f}(\hat{x}) \hat{g}(\hat{x})|0\rangle .
$$

We emphasize that the star product depends on the realization $\Phi_{\mu \nu}$. The result relating the star product and the coproduct obtained for non-covariant realizations 22. can be extended to covariant realizations of $\kappa$-deformed space, Eq. (11), as

$$
(f \star g)(u)=\left.m\left(e^{u_{\alpha}\left(\triangle-\triangle_{0}\right) \partial_{\alpha}} f(x) \otimes g(y)\right)\right|_{\substack{x=u \\ y=u}},
$$

where $m$ is the multiplication map in the Hopf algebra and $\triangle_{0} \partial_{\mu}=\partial_{\mu} \otimes \mathbf{1}+\mathbf{1} \otimes \partial_{\mu}$ is the undeformed coproduct.

\subsection{Star product for the realization of type I}

In this subsection we discuss the star product in the realization of type I assuming that the parameter function $\varphi$ depends only on the variable $A=i a \partial$. In view of Eq. (61), we have

$$
\hat{x}_{\mu}=x_{\mu} \varphi(A)+i(x \partial) a_{\mu} \gamma(A)
$$

where

$$
\gamma(A)=\frac{1+\varphi^{\prime}(A)}{1-A \frac{\varphi^{\prime}(A)}{\varphi(A)}}, \quad \varphi(0)=1 .
$$

In order to find the coproduct $\triangle \partial_{\mu}$, we note that in this realization $\partial_{\mu}=\varphi(A) \partial_{\mu}^{R}$. The coproduct for $\partial_{\mu}^{R}$ is given by Eq. (34), hence

$$
\begin{aligned}
\triangle \partial_{\mu} & =\triangle \varphi(A) \triangle \partial_{\mu}^{R} \\
& =\triangle \varphi(A)\left[\frac{1}{\varphi(A)} \partial_{\mu} \otimes \mathbf{1}+Z \otimes \frac{1}{\varphi(A)} \partial_{\mu}\right] .
\end{aligned}
$$


Inverting the expression for the shift operator $Z=1+A / \varphi(A)$, we find

$$
A=(Z-1)+\varphi^{\prime}(0)(Z-1)^{2}+\cdots
$$

which, together with $\triangle Z=Z \otimes Z$, allows us to calculate the coproduct $\triangle \varphi(A)$. Then, to second order in the parameter $a$, Eq. (119) leads to

$$
\begin{aligned}
\triangle \partial_{\mu} & =\partial_{\mu}^{x}\left[1+\varphi^{\prime}(0) A_{y}+\left(\varphi^{\prime \prime}(0)+\left(\varphi^{\prime}(0)\right)^{2}+\varphi^{\prime}(0)\right) A_{x} A_{y}+\frac{1}{2} \varphi^{\prime \prime}(0) A_{y}^{2}\right] \\
& +\partial_{\mu}^{y}\left[1+\left(1+\varphi^{\prime}(0)\right) A_{x}+\left(\varphi^{\prime \prime}(0)+\left(\varphi^{\prime}(0)\right)^{2}+\varphi^{\prime}(0)\right) A_{x} A_{y}+\frac{1}{2} \varphi^{\prime \prime}(0) A_{x}^{2}\right],
\end{aligned}
$$

where $A_{x}=i a \partial^{x}, A_{y}=i a \partial^{y}$. Consequently, the star product from Eq. (116) is given by

$$
\begin{aligned}
(f \star g)(u) & =\left\{1+u \partial^{x}\left[\varphi^{\prime}(0) A_{y}+\left(\varphi^{\prime \prime}(0)+\left(\varphi^{\prime}(0)\right)^{2}+\varphi^{\prime}(0)\right) A_{x} A_{y}+\frac{1}{2} \varphi^{\prime \prime}(0) A_{y}^{2}\right]\right. \\
& +u \partial_{y}\left[\left(1+\varphi^{\prime}(0)\right) A_{x}+\left(\varphi^{\prime \prime}(0)+\left(\varphi^{\prime}(0)\right)^{2}+\varphi^{\prime}(0)\right) A_{x} A_{y}+\frac{1}{2} \varphi^{\prime \prime}(0) A_{x}\right] \\
& \left.+\frac{1}{2}\left[\varphi^{\prime}(0) u \partial^{x} A_{y}+\left(1+\varphi^{\prime}(0)\right) u \partial^{y} A_{x}\right]^{2}\right\}\left.f(x) g(y)\right|_{\substack{x=u \\
y=u}} .
\end{aligned}
$$

One can show that the dual relation holds

$$
(f \star g)_{\varphi(A)}=(g \star f)_{\varphi(-A)-A}
$$

where the star products correspond to the functions $\varphi(A)$ and $\varphi(-A)-A$, respectively.

\subsection{Star product for special realizations}

In this subsection we give the star products in closed form for the left, right and symmetric realizations, as well as the star product to second order in $a$ in the natural realization.

Left realization: $\varphi_{L}=1-A$.

$$
(f \star g)_{\varphi_{L}}(u)=\left.e^{-u_{\alpha} \partial_{\alpha}^{x} A_{y}} f(x) g(y)\right|_{\substack{x=u \\ y=u}} .
$$


Right realization: $\varphi_{R}=1$.

$$
(f \star g)_{\varphi_{R}}(u)=\left.e^{u_{\alpha} \partial_{\alpha}^{y} A_{x}} f(x) g(y)\right|_{\substack{x=u \\ y=u}} .
$$

The "left" and "right" star products satisfy the symmetry relation

$$
(f \star g)_{\varphi_{L}}=(g \star f)_{\varphi_{R}}
$$

Symmetric realization: $\varphi_{S}=A /(\exp (A)-1)$.

$$
(f \star g)_{\varphi_{S}}(u)=\left.e^{u_{\alpha}\left(\triangle-\triangle_{0}\right) \partial_{\alpha}} f(x) g(y)\right|_{\substack{x=u \\ y=u}},
$$

where

$$
\begin{aligned}
\triangle_{0} \partial_{\mu} & =\partial_{\mu}^{x}+\partial_{\mu}^{y} \\
\triangle \partial_{\mu} & =\partial_{\mu}^{x} \frac{\varphi_{S}\left(A_{x}+A_{y}\right)}{\varphi_{S}\left(A_{x}\right)}+\partial_{\mu}^{y} \frac{\varphi_{S}\left(-A_{x}-A_{y}\right)}{\varphi_{S}\left(-A_{y}\right)} .
\end{aligned}
$$

In this case, we have

$$
(f \star g)_{\varphi_{S}(A)}=(g \star f)_{\varphi_{S}(-A)} .
$$

The symmetric realization $\varphi=\varphi_{S}$ corresponds to the symmetric Weyl ordering [22]. Our closed form results, Eqs. (127) and (129), are in agreement with the general series expansion formula for the star product of the Lie algebra type NC space 42 .

Natural realization: $\varphi_{N}=-A+\sqrt{1-B}$.

In this realization we have $\partial_{\mu}=D_{\mu}$, hence the coproduct $\triangle \partial_{\mu}$ is given by Eq. (27). One can show that to second order in the parameter $a$ the star product yields

$$
\begin{aligned}
(f \star g)_{\varphi_{N}}(u) & =f(u) g(u) \\
& +\left\{u_{\mu}\left[\left(-\partial_{\mu}^{x}-\frac{i a_{\mu}}{2 a^{2}} a_{\alpha}^{2}\left(\partial_{\alpha}^{x}\right)^{2}\right) A_{y}-\frac{1}{2} \partial_{\mu}^{x} a_{\alpha}^{2}\left(\partial_{\alpha}^{y}\right)^{2}+i a_{\mu}\left(1+A_{x}\right) \partial^{x} \partial^{y}\right]\right. \\
& \left.+\frac{1}{2} u_{\mu} u_{\nu}\left[\partial_{\mu}^{x} \partial_{\nu}^{y} A_{y}^{2}-2 i a_{\mu} \partial_{\nu}^{x} \partial^{x} \partial^{y} A_{y}-a_{\mu} a_{\nu}\left(\partial^{x} \partial^{y}\right)^{2}\right]\right\}\left.f(x) g(y)\right|_{\substack{x=u \\
y=u}},
\end{aligned}
$$

where $\partial^{x} \partial^{y}=\partial_{\alpha}^{x} \partial_{\alpha}^{y}$. 


\subsection{Equivalent star products}

So far we have considered the star product for some specific realizations of type I and II. We point out, however, that infinitely many realizations of the star product can be constructed by similarity transformations of the variables $x_{\mu}, \partial_{\mu}$. In the following we consider the star products obtained by similarity transformations starting from the right realization.

Recall that in the right realization we have

$$
\hat{x}_{\mu}=x_{\mu}^{R}+i a_{\mu}\left(x^{R} \partial^{R}\right),
$$

and the star product is given by Eq. (125). (From now on the variables $x_{\mu}$ and $\partial_{\mu}$ used in the right realization will be denoted by $x_{\mu}^{R}$ and $\partial_{\mu}^{R}$, respectively.) A similarity transformation is defined by

$$
\begin{aligned}
& x_{\mu}=S^{-1} x_{\mu}^{R} S, \\
& \partial_{\mu}=S^{-1} \partial_{\mu}^{R} S .
\end{aligned}
$$

Clearly, the new variables $x_{\mu}$ and $\partial_{\mu}$ also generate the Heisenberg algebra. We define the vacuum condition on $S$ by $S|0\rangle=|0\rangle$. In view of Eq. (125), the star product induced by the similarity transformation $S$ is given by

$$
\begin{aligned}
(f \underset{S}{\star} g)(u) & =S\left(S^{-1} f \star S^{-1} g\right)_{\varphi_{R}}(u) \\
& =\left.S e^{u_{\alpha} \partial_{\alpha}^{y} A_{x}}\left(S^{-1} f\right)(x)\left(S^{-1} g\right)(y)\right|_{\substack{x=u \\
y=u}} .
\end{aligned}
$$

Two star products are said to be equivalent if they are related by a similarity transformation. For example, if $S=e^{-x \partial A_{x}}$, then

$$
(f \star g)_{\varphi_{L}}(u)=S\left(S^{-1} f \star S^{-1} g\right)_{\varphi_{R}}(u),
$$

hence the star products for the left and right realization are equivalent. We will show that all realizations of type I with $\varphi=\varphi(A)$ lie in the orbits of the action of similarity transformations of the right realization. Hence, any two star products in realizations of this type are equivalent.

Consider a realization of type I,

$$
\hat{x}_{\mu}=x_{\mu} \varphi(A)+i a_{\mu}(x \partial) \frac{1+\varphi^{\prime}(A)}{1-A \frac{\varphi^{\prime}(A)}{\varphi(A)}}, \quad \varphi(0)=1 .
$$


The transformation $\left(x_{\mu}, \partial_{\mu}\right) \mapsto\left(x_{\mu}^{R}, \partial_{\mu}^{R}\right)$ which maps the realization (132) into (137) is given by

$$
\begin{aligned}
& x_{\mu}^{R}=x_{\mu} \varphi(A)+i a_{\mu}(x \partial) \frac{\varphi^{\prime}(A)}{1-A \frac{\varphi^{\prime}(A)}{\varphi(A)}}, \\
& \partial_{\mu}^{R}=\partial_{\mu} \frac{1}{\varphi(A)} .
\end{aligned}
$$

We show that there exists a similarity operator of the form $S=\exp (U)$,

$$
U=(x \partial) \sum_{k=1}^{\infty} c_{k} A^{k},
$$

such that Eqs. (138)-(139) are given by $x_{\mu}^{R}=S x_{\mu} S^{-1}$ and $\partial_{\mu}^{R}=S \partial_{\mu} S^{-1}$, respectively. Then, Eq. (139) yields

$$
\exp (\operatorname{ad}(U)) \partial_{\mu}=\partial_{\mu} \frac{1}{\varphi(A)}
$$

By expanding both sides of Eq. (141) into power series in $A$, one can show that the coefficients $c_{k}$ are uniquely determined by the function $\varphi(A)$. Expanding the right-hand side of Eq. (141) leads to

$$
\partial_{\mu} \frac{1}{\varphi(A)}=\partial_{\mu}\left(1+\sum_{p=1}^{\infty} \frac{1}{p !} \varepsilon_{p} A^{p}\right),
$$

where the coeffcients $\varepsilon_{p}$ can be found recursively from

$$
\begin{aligned}
& \varepsilon_{1}=-\varphi^{\prime}(0), \\
& \varepsilon_{p}=-\varphi^{(p)}(0)-\sum_{k=1}^{p-1}\left(\begin{array}{l}
p \\
k
\end{array}\right) \varphi^{(k)}(0) \varepsilon_{p-k}, \quad p \geq 2 .
\end{aligned}
$$

Similarly, the expansion of the left-hand side of Eq. (141) gives

$$
\exp (\operatorname{ad}(U)) \partial_{\mu}=\partial_{\mu}\left(1+\sum_{p=1}^{\infty} \gamma_{p} A^{p}\right)
$$

where the coefficients $\gamma_{p}$ are found in terms of $c_{k}$ as follows:

$$
\begin{aligned}
& \gamma_{1}=-c_{1}, \\
& \gamma_{p}=-c_{p}+\sum_{n=2}^{p} \frac{(-1)^{n}}{n !} \beta_{p}^{(n)}, \quad p \geq 2 .
\end{aligned}
$$


Here,

$$
\beta_{p}^{(n)}=\sum_{|k|=p} \Psi(k) \prod_{i=1}^{n} c_{k_{i}}
$$

where $k=\left(k_{1}, k_{2}, \ldots, k_{n}\right), k_{i} \in \mathbb{N}$, is a multi-index with length $|k|=\sum_{i=1}^{n} k_{i}$, the function $\Psi(k)$ is defined by

$$
\Psi(k)=\left(1+k_{n}\right)\left(1+k_{n}+k_{n-1}\right) \ldots\left(1+\sum_{k=2}^{n} k_{i}\right),
$$

and the summation is taken over all multi-indices such that $|k|=p$. It follows from Eqs. (142) and (145) that

$$
\begin{aligned}
& c_{1}=-\varepsilon_{1}, \\
& c_{p}=\sum_{n=2}^{p} \frac{(-1)^{n}}{n !} \beta_{p}^{(n)}-\frac{1}{p !} \varepsilon_{p}, \quad p \geq 2 .
\end{aligned}
$$

Note that the coefficient $c_{p}$ is uniquely determined by $c_{1}, c_{2}, \ldots, c_{p-1}$. Hence, Eqs. (150)-(151), together with Eqs. (143)-(144), provide recursion relations for $c_{p}$ in terms of $\varphi^{(k)}(0)$, thus $S$ is uniquely determined by $\varphi(A)$. For example, the first three coefficients are given by $c_{1}=\varphi^{\prime}(0), c_{2}=\frac{1}{2} \varphi^{\prime \prime}(0), c_{3}=\frac{1}{6} \varphi^{\prime \prime \prime}(0)+\frac{1}{4} \varphi^{\prime}(0) \varphi^{\prime \prime}(0)$. Equations (135) and (140), together with $c_{1}$ and $c_{2}$, reproduce the star product given by Eq. (122). This represents an important consistency check of our approach.

Note that our covariant realizations for $\hat{x}_{\mu}$, Eq. (47), and for $D_{\mu}$, Eq. (54), follow from $S=e^{U}$, where

$$
U=(x \partial) \Phi_{1}(A, B)+(x a) \partial^{2} \Phi_{2}(A, B),
$$

with boundary conditions $\Phi_{1}(0,0)=0$ and $\Phi_{2}(0,0)$ finite.

\subsection{Scalar fields and invariants on kappa-deformed space}

All covariant realizations are physically equivalent. Here we consider the natural realization $x^{N}$, $\partial^{N}$ defined by $D_{\mu}=\partial_{\mu}^{N}$ and $M_{\mu \nu}=x_{\mu}^{N} \partial_{\nu}^{N}-x_{\nu}^{N} \partial_{\mu}^{N}$ (c.f. Sects. 3.1 and 4.2). The realization of the NC coordinates is given by Eq. (93),

$$
\hat{x}_{\mu}=x_{\mu}^{N} Z^{-1}+i\left(a x^{N}\right) \partial_{\mu}^{N}
$$


Let us consider a scalar field $\hat{\Phi}(\hat{x})$ in the NC coordinates satisfying $\left[M_{\mu \nu}, \hat{\Phi}(\hat{x})\right]=0$ for all $\mu, \nu$. Then we define a scalar field $\Phi\left(x^{N}\right)$ in the undeformed space by

$$
\hat{\Phi}\left(\hat{x}\left(x^{N}\right)\right)|0\rangle=\Phi\left(x^{N}\right) .
$$

The ordinary Fourier transform is defined by

$$
\tilde{\Phi}(k)=\int d^{n} x^{N} e^{-i k x^{N}} \Phi\left(x^{N}\right), \quad k \in \mathbb{R}^{n} .
$$

Then using the relation (111),

$$
e^{i k \hat{x}}|0\rangle=e^{i P_{N}(k) x^{N}}
$$

where $P_{N}(k)$ is given by Eq. (112), we find

$$
\hat{\Phi}(\hat{x})=\int d^{n} k \tilde{\Phi}(k) e^{i P_{N}^{-1}(k) \hat{x}}
$$

which holds in any realization of $\hat{x}$. Here $P_{N}^{-1}$ denotes the inverse function of $P_{N}$,

$$
P_{N}^{-1}(k)_{\mu}=\frac{\ln Z^{-1}(k)}{Z^{-1}(k)-1}\left(k_{\mu}+\frac{a_{\mu}}{a^{2}}\left(\sqrt{1+a^{2} k^{2}}-1\right)\right),
$$

where

$$
Z^{-1}(k)=\sqrt{1+a^{2} k^{2}}+a k .
$$

The above relation (157) represents a construction of $S O_{a}(n)$ invariants $\hat{\Phi}(\hat{x})$ in terms of $\Phi\left(x^{N}\right)$ and $\tilde{\Phi}(k)$ by using the natural realization (93). Alternatively, from Eq. (93) we obtain the inverse mapping

$$
x_{\mu}^{N}=\left(\hat{x}_{\mu}-i(a \hat{x}) \frac{\partial_{\mu}^{N}}{\sqrt{1-a^{2}\left(\partial_{\mu}^{N}\right)^{2}}}\right) Z .
$$

Then, we find

$$
\Phi\left(x^{N}(\hat{x})\right)|0\rangle=\hat{\Phi}(\hat{x}) .
$$

Both constructions are consistent and equivalent. Furthermore, we define the invariant integration over the entire NC space using the natural realization as follows:

$$
\int \hat{\Phi}(\hat{x})=\int d^{n} x^{N} \Phi\left(x^{N}\right)
$$


with the property

$$
\int \hat{\Phi}_{1}(\hat{x}) \hat{\Phi}_{2}(\hat{x})=\int d^{n} x^{N}\left(\Phi_{1} \star \Phi_{2}\right)_{N}\left(x^{N}\right) .
$$

A generalized action of the scalar field $\Phi(\hat{x})$ on the NC $\kappa$-deformed space is simply the action of the ordinary scalar field in natural coordinates $x^{N}$ on the undeformed space in which pointwise multiplication of fields is replazed by $\star$-mulitplication in the natural realization. Further inverstigation of this problem is in progress and will be published separately.

\section{Conclusion}

We have constructed covariant realizations of a general $\kappa$-deformed space in terms of commutative coordinates $x_{\mu}$ and their derivatives $\partial_{\mu}$ in the undeformed space. Our construction can also be applied to spaces with arbitrary signatures, especially to Minkowski-type spaces.

Particulary, we have studied $\kappa$-deformed space whose deformation is described by an arbitrary vector. The NC coordinates and rotation generators form an extended Lie algebra. The subalgebra of rotation generators, $S O_{a}(n)$, is undeformed. The Dirac derivatives mutually commute and are vector-like under the action of $S O_{a}(n)$. By introducing the shift operator the deformed Heisenberg algebra is written in a very simple way. We have presented the Leibniz rule and coproduct for the rotation generators $M_{\mu \nu}$ and the Dirac derivatives $D_{\mu}$ in a covariant form.

We have found two types of covariant realizations which are described by an arbitrary function $\varphi(A)$ with $\varphi(0)=1$. We point out that all covariant realizations are equivalent and on an equal footing. We have constructed coproducts and star products for covariant realizations. There is an important relation between the coproduct and the star product in terms of an exponential map for a given realization. Specially, we have found a few realizations (covariant left, right, symmetric and natural) which have very simple properties. All realizations of type I are related by similarity transformations and the corresponding star products are equivalent. We have considered scalar fields in NC coordinates and demonstrated their simple properties using the natural realization. Constructions of invariants and invariant integration on NC spaces are also discussed. 
Our approach may be useful in quantum gravity models, specially in $2+1$ dimensions. In this case, the coresponding Lie algebra is $S U(2)$ or $S U(1,1)$ [43], [44], 45]. It would be interesting to classify $\mathrm{NC}$ spaces with covariant realizations in which $\mathrm{NC}$ coordinates and rotation generators form an extended Lie algebra. For example, Snyder space is of this type and covariant realizations in terms of undeformed space exist [46].

\section{Acknowledgments}

One of us (S.M.) thanks D. Svrtan and Z. Škoda for useful discussions. This work is supported by the Ministry of Science and Technology of the Republic of Croatia under the contracts 098-0000000-2865 and 0023003.

\section{References}

[1] S. Doplicher, K. Fredenhagen and J. E. Roberts, The quantum structure of spacetime at the Planck scale and quantum fields, Phys. Lett. B 331, 39 (1994); Commun. Math. Phys. 172, 187 (1995) hep-th/0303037.

[2] N. Seiberg and E. Witten, String theory and noncommutative geometry, JHEP 09, 032 (1999) hep-th/9908142;

J. de Boer, P. A. Grassi and P. van Nieuwenhuizen, Noncommutative superspace from string theory, Phys. Lett. B 574, 98 (2003) |hep-th/0302078|.

[3] M. R. Douglas and N. A. Nekrasov, Noncommutative field theory, Rev. Mod. Phys. 73, 977 (2001) hep-th/0106048.

[4] R. J. Szabo, Quantum field theory on noncommutative spaces, Phys. Rept. 378, 207 (2003) hep-th/0109162;

R. J. Szabo, Symmetry, gravity and noncommutativity, Class. Quant. Grav. 23 (2006) R199-R242, hep-th/0606233.

[5] P. Aschieri, B. Jurco, P. Schupp and J. Wess, Noncommutative GUTs, standard model and C,P,T; Nucl. Phys. B 651, 45 (2003) hep-th/0205214;

P. Aschieri, C. Blohmann, M. Dimitrijević, F. Meyer, P. Schupp and J. Wess, A 
gravity theory on noncommutative spaces, Class. Quant. Grav. 22, 3511 (2005) hep-th/0504183.

[6] J. Lukierski, A. Nowicki, H. Ruegg and V. N. Tolstoy, Q-deformation of Poincaré algebra, Phys. Lett. B 264, 331 (1991).

[7] J. Lukierski, A. Nowicki and H. Ruegg, New quantum Poincaré algebra, and $\kappa$-deformed field theory, Phys. Lett. B 293, 344 (1992).

[8] J. Lukierski and H. Ruegg, Quantum $\kappa$-Poincaré in any dimension, Phys. Lett. B 329, 189 (1994) hep-th/9310117.

[9] S. Majid and H. Ruegg, Bicrossproduct structure of $\kappa$-Poincaré group and noncommutative geometry, Phys. Lett. B 334, 348 (1994) hep-th/9404107.

[10] P. Kosiński and P. Maślanka, The duality between $\kappa$-Poincaré algebra and $\kappa$ Poincaré group, [ hep-th/9411033].

[11] A. Sitarz, Noncommutative differential calculus on the kappa-Minkowski space, Phys. Lett. B 349, 42 (1995) [hep-th/9409014].

[12] K. Kosiński, J. Lukierski and P. Maślanka, Local $D=4$ field theory on $\kappa$ Minkowski space, Phys. Rev. D 62, 025004 (2000) [hep-th/9902037].

[13] K. Kosiński, J. Lukierski and P. Maślanka, Local field theory on $\kappa$-Minkowski space, «-products and noncommutative translations, Czech. J. Phys. 50, 1283 (2000) hep-th/0009120.

[14] P. Kosiński, J. Lukierski, P. Maślanka and A. Sitarz, Generalised $\kappa$ deformations and deformed relativistic scalar fields on noncommutative Minkovski space, hep-th/0307038.

[15] G. Amelino-Camelia, Testable scenario for relativity with minimum-length, Phys. Lett. 510, 255 (2001) hep-th/0012238;

G. Amelino-Camelia, Relativity in space-times with short-distance structure governed by an observer-independent (Planckian) length scale, Int. J. Mod. Phys. D 11, 35 (2002) [gr-qc/0012051];

N. R. Bruno, G. Amelino-Camelia and J. Kowalski-Glikman, Deformed boost 
transformations that saturate at the Planck scale, Phys. Lett. B 522, 133 (2001) hep-th/0107039].

[16] J. Kowalski-Glikman and S. Nowak, Double special relativity theories as different bases of kappa-Poincaré algebra, Phys. lett. B 539, 126 (2002) hep-th/0203040].

[17] G. Amelino-Camelia and M. Arzano, Coproduct and star-product in field theories on Lie algebra noncomutative spacetime, Phys. Rev. D 65, 084044 (2002) hep-th/0105120].

[18] G. Amelino-Camelia, F. D'Andrea and G. Mandanici, Group velocity in noncommutative spacetime, JCAP 0309 (2003) 006 [hep-th/0211022].

[19] M. Dimitrijević, L. Jonke, L. Möller, E. Tsouchnika, J. Wess and M. Wohlgenannt, Deformed field theory on $\kappa$-spacetime, Eur. Phys. C 31, 129 (2003) hep-th/0307149.

[20] M. Dimitrijević, F. Meyer, L. Möller and J. Wess, Gauge theories on the $\kappa$ Minkovski space time, Eur. Phys. J. C 36, 117 (2004) hep-th/0310116.

[21] M. Dimitrijević, L. Möller and E. Tsouchnika, Derivatives, forms and vector fields on the $\kappa$-deformed Euclidean space, J. Phys. A 37 (2004) hep-th/0404224.

[22] S. Meljanac and M. Stojić, New realizations of Lie algebra kappa-deformed Euclidean space, Eur. Phys. J. C 47, 531 (2006) [hep-th/0605133.

[23] L. Möller, A symmetry invariant integral on $\kappa$-deformed spacetime, JHEP 0512, 029 (2005) hep-th/0409128;

A. Agostini, G. Amelino-Camelia, M. Arzano and F. D'Andrea, Action functional for $\kappa$-Minkowski noncommutative spacetime, hep-th/0407227.

[24] A. Agostini, G. Amelino-Camelia, M. Arzano, A. Marciano and R.A. Tacchi, Generalizing the Noether theorem for Hopf-algebra spacetime symmetries, hep-th/0607221. 
[25] L. Freidel, J. Kowalski-Glikman and S. Nowak, ¿From noncommutative kappaMinkowski to Minkowski space-time, hep-th/0612170.

[26] S. Ghosh, The AdS particle, Phys. Lett. B 623, 251 (2005) hep-th/0506084;

S. Ghosh and P. Pal, Kappa-Minkowski spacetime through exotic "oscillator", Phys. Lett. B 618, 243 (2005) hep-th/0502192.

[27] S. Ghosh, A Lagrangian for DSR particle and the role of noncommutativity, Phys. Rev. D 74, 084019 (2006) hep-th/0608206.

[28] Hyeong-Chan Kim, Jae Hyung Yee and Chaiho Rim, Kappa-Minkowski spacetime and a uniformly accelerating observer, hep-th/0701054.

[29] D. Bonatsos and C. Daskaloyannis, General deformation schemes and $N=2$ supersymmetric quantum mechanics, Phys. Lett. B 307, 100 (1993);

S. Meljanac, M. Mileković and S. Pallua, Unified view of deformed single-mode oscillator algebras, Phys. Lett. B 328, 55 (1994) hep-th/9404039.

[30] S. Meljanac and M. Mileković, Unified view of multimode algebras with Focklike representation, Int. J. Mod. Phys. A 11, 1391 (1996).

[31] S. Meljanac and A. Perica, Number operators in a general quon algebra, J. Phys. A: Math. Gen. 27, 4737 (1994);

S. Meljanac and A. Perica, Generalized quon statistics, Mod. Phys. Lett. A 9, 3293 (1994) hep-th/9409180;

S. Meljanac, A. Perica and D. Svrtan, The energy operator for a model with a multiparametric infinite statistics, J. Phys. A 36, 6337 (2003) math-ph/0304038.

[32] S. Meljanac and D. Svrtan, Determinants and inversion of Gramm matrices in Fock representation of $q_{k l}$-canonical commutation relations and applications to hyperplane arrangements and quantum groups. Proof of an extension of Zagier's conjecture, math-ph/0304040];

S. Meljanac and D. Svrtan, Study of Gramm matrices in Fock representation of multiparametric canonical commutation relations, extended Zagier's conjecture, hyperplane arrangements and quantum group, Math. Commum. 1 (1996) no.1, 1-24 math-ph/0304039. 
[33] V. Bardek and S. Meljanac, Deformed Heisenberg algebras, a Fock space representation and the Calogero model, Eur. Phys. J. C 17, 539 (2000) hep-th/0009099];

V. Bardek, L. Jonke, S. Meljanac and M. Mileković, Calogero model, deformed oscillators and the collapse, Phys. Lett. B 531, 311 (2002) hep-th/0107053.

[34] S. Meljanac, M. Mileković and M. Stojić, Permutation invariant algebras, a Fock space realization and the Calogero model, Eur. Phys. J. C 24, 331 (2002) math-ph/0201061].

[35] L. Jonke and S. Meljanac, Bosonic realization of algebras in the Calogero model, Phys. Lett. B 526, 149 (2002) hep-th/0106135.

[36] V. P. Nair and A. P. Polychronakos, Quantum mechanics on the noncommutative plane and sphere, Phys. Lett. B 505, 267 (2001) [hep-th/0011172];

L. Jonke and S. Meljanac, Representations of noncommutative quantum mechanics and symmerties, Eur. Phys. J. C 29, 433 (2003) [hep-th/0210042];

I. Dadić, L. Jonke and S. Meljanac, Harmonic oscillator on noncommutative spaces, Acta Phys. Slovaca 55, 149 (2005) |hep-th/0301066|.

[37] A. Kempf, G. Mangano and R.B. Mann, Hilbert space representation of the minimal length uncertainty relation, Phys. Rev. D 52, 1108 (1995) hep-th/9412167;

L. N. Chang, D. Minic, N. Okamura and T. Takeuchi, The effect of the minimal length uncertainty relation on the density of states and the cosmological constant problem, Phys. Rev. D 65, 125027 (2002) [hep-th/0201017);

I. Dadić, L. Jonke and S. Meljanac, Harmonic oscillator with minimal length uncertainty relations and ladder operators, Phys. Rev. D 67, 087701 (2003) hep-th/0210264].

[38] J. Wess, Deformed coordinates spaces; Derivatives, Lecture given at the Balkan workshop BW2003, August 2003, Vrnjačka Banja, Serbia, [hep-th/0408080].

[39] M. Chaichian, P. P. Kulish, K. Nishijima and A. Tureanu, On a Lorentzinvariant interpretation on noncommutative space-time and its implications on noncommutative QFT, Phys. Lett. B 604, 98 (2004) [hep-th/0408069]; 
M. Chaichian, P. Presnajder and A. Tureanu, New concept of relativistic invariance in NC space-time: Twisted Poincaré symmetry and its implications, Phys. Rev. Lett. 94, 151602 (2005) hep-th/0409096.

[40] J. Lukierski, M. Woronowicz, New Lie algebraic and quadratic deformations of Minkowski space from twisted Poincaré symmetries, Phys. Lett. B 633, 116 (2006) hep-th/0508083.

[41] N. Durov, S. Meljanac, A. Samsarov and Z. Škoda, A universal formula for representing Lie algebra generators as formal power series with coefficients in the Weyl algebra, J. Algebra 309 (2007) [math.RT/0604096].

[42] V. Kathotia, Kontsevich's universal formula for deformation quantisation and the Campbell-Baker-Hausdorff formula, Internat. J. Math. 11, 523 (2000), no. 4 math.qa/9811174.

[43] L. Freidel and E. R. Livine, Ponzano-Regge model revisited III: Feynman diagrams and effective field theory, Class. Quant. Grav. 23, 2021 (2006) hep-th/0502106];

L. Freidel and E. R. Livine, 3d quantum gravity and effective noncommutative quantum field theory, Phys. Rev Lett. 96, 221301 (2006) hep-th/0512113.

[44] S. Majid, Noncommutative model with spontaneous time generator and Planckian bound, J. Math. Phys. 46103520 (2005) [hep-th/0507271].

[45] L. Freidel and S. Majid, Noncommutative harmonic analysis, sampling theory and the Duflo map in 2+1 quantum gravity, [hep/th-0601004].

[46] R. Banerjee, S. Kulkarni and S. Samatra, Deformed symmetry in Snyder space and relativistic particle dynamics, JHEP 0605, 077 (2006) [hep-th/0602151]. 